This is an electronic reprint of the original article. This reprint may differ from the original in pagination and typographic detail.
Author(s): Volchenko, Vladimir; Akhrameev, Evgeniy; Bezrukov, Leonid; Dzaparova, Irina; Davitashvili, Irakliy; Enqvist, Timo; Fynbo, Hans; Guliev, Zhamal; Inzhechik, Lev; Izmaylov, Alexander; Joutsenvaara, Jari; Khabibullin, Marat; Khotjantsev, Alexey; Kudenko, Yuri; Kuusiniemi, Pasi; Lubsandorzhiev, Bayarto; Lubsandorzhiev, Nima; Mineev, Oleg; Olanterä, Lauri; Petkov, Valeriy; Poleshuk, Roman; Räihä, Tomi;
Title: $\quad$ Background and muon counting rates in underground muon measurements with a plastic scintillator counter based on a wavelength shifting fibre and a multi-pixel avalanche photodiode readout

Year: $\quad 2010$

Version:

Please cite the original version:

Volchenko, V., Akhrameev, E., Bezrukov, L., Dzaparova, I., Davitashvili, I., Enqvist, T., Fynbo, H., Guliev, Z., Inzhechik, L., Izmaylov, A., Joutsenvaara, J., Khabibullin, M., Khotjantsev, A., Kudenko, Y., Kuusiniemi, P., Lubsandorzhiev, B., Lubsandorzhiev, N., Mineev, O., Olanterä, L., ... Yershov, N. (2010). Background and muon counting rates in underground muon measurements with a plastic scintillator counter based on a wavelength shifting fibre and a multi-pixel avalanche photodiode readout. Central European Journal of Physics, 8(3), 445-447. https://doi.org/10.2478/s11534009-0135-4

All material supplied via JYX is protected by copyright and other intellectual property rights, and duplication or sale of all or part of any of the repository collections is not permitted, except that material may be duplicated by you for your research use or educational purposes in electronic or print form. You must obtain permission for any other use. Electronic or print copies may not be offered, whether for sale or otherwise to anyone who is not an authorised user. 


\title{
Background and muon counting rates in underground muon measurements with a plastic scintillator counter based on a wavelength shifting fibre and a multi-pixel avalanche photodiode readout
}

Vladimir I. Volchenko ${ }^{1}$, Evgeniy V. Akhrameev ${ }^{1}$, Leonid B. Bezrukov ${ }^{1}$, Irina M. Dzaparova ${ }^{1}$, Irakliy Sh. Davitashvili ${ }^{1}$, Timo Enqvist ${ }^{2}$, Hans Fynbo ${ }^{4}$, Zhamal Sh. Guliev ${ }^{1}$, Lev V. Inzhechik ${ }^{1}$, Alexander O. Izmaylov ${ }^{1}$, Jari Joutsenvaara ${ }^{2}$, Marat M. Khabibullin ${ }^{1}$, Alexey N. Khotjantsev ${ }^{1}$, Yuri G. Kudenko ${ }^{1}$, Pasi Kuusiniemi ${ }^{2}$, Bayarto K. Lubsandorzhiev ${ }^{1 *}$, Nima B. Lubsandorzhiev${ }^{1}$, Oleg V. Mineev ${ }^{1}$, Lauri Olanterä ${ }^{2}$, Valeriy B. Petkov ${ }^{1}$, Roman V. Poleshuk ${ }^{1}$, Tomi Räihä ${ }^{2}$, Bator A. M. Shaibonov ${ }^{1}$, Juho Sarkamo ${ }^{2}$, Alexey T. Shaykhiev ${ }^{1}$, Wladyslaw Trzaska ${ }^{3}$, Galina V. Volchenko ${ }^{1}$, Alexey F. Yanin ${ }^{1}$, Nikolay V. Yershov ${ }^{1}$

1 Institute for Nuclear Research of RAS, Moscow Russia

2 CUPP/Pyhäsalmi, University of Oulu, Oulu Finland

3 Department of Physics, University of Jyväskylä, Jyväskylä Finland

4 Department of Physics and Astronomy, University of Århus, Denmark

Received 23 April 2009; accepted 19 August 2009

\begin{abstract}
:
In this short note we present results of background measurements carried out with polystyrene based cast plastic $12.0 \times 12.0 \times 3.0 \mathrm{~cm}^{3}$ size scintillator counter with a wavelength shifting fibre and a multi-pixel Geiger mode avalanche photodiode readout in the Baksan underground laboratory at a depth of 200 metres of water equivalent. The total counting rate of the scintillator counter measured at this depth and at a threshold corresponding to $\sim 0.37$ of a minimum ionizing particle is approximately $1.3 \mathrm{~Hz}$.
\end{abstract}

PACS (2008): 29.40.Mc; 85.60.Dw; 95.85.Ry

Keywords: plastic scintillator $\cdot \operatorname{cosmic}$ ray $\cdot$ muon $\cdot$ radioactivity background $\cdot$ multi-pixel avalanche photodiode

(c) Versita Sp. z o.o.

\section{Introduction}

*E-mail: lubsand@pcbai10.inr.ruhep.ru,

lubsand@pit.physik.uni-tuebingen.de

Plastic scintillator counters are presently widely used in many underground experiments as scintillator muon- 
veto counters in neutrinoless double beta-decay exper-

iments (e.g. GERDA ${ }^{1}$ ), dark matter search experiments (e.g. CRESST-II [1]) and cosmic ray muon experiments like EMMA $[2,3]$. A scintillator counter is usually a plastic scintillator plate which is thick enough in order to have a good amplitude response to cosmic ray muons or minimum ionizing particles (MIP). In all such experiments the main problem is how to reach high efficiency for cosmic ray muon detection while at the same time to provide high efficiency of background rejection. The background is mainly caused by radioactive decays in the rock surrounding the detector. The background spectrum lies in a lower energy domain in comparison to muon events but has a rather long tail overlapping with the muon spectrum. Thus one should set the energy threshold such that both muon detection efficiency and background rejection are at an acceptable level for the experiment. This can be carried out if there is a good separation between muon and background events. Energy deposition of a relativistic muon in plastic scintillator depends only on the scintillator thickness. One solution is to use thicker scintillator plates.

\section{Experiment}

We have carried out a dedicated measurement of the background counting rate and muon detection efficiency at a depth of 200 metres of water equivalent (m.w.e.) in the Baksan underground laboratory using a small plastic scintillator counter manufactured for the cosmic ray muon experiment EMMA at Pyhäsalmi mine in Finland $[2,3]$.

A schematic view of the scintillator counter is shown in Fig. 1. The scintillator counter is a polystyrene based cast plastic scintillator of $12.0 \times 12.0 \times 3.0 \mathrm{~cm}^{3}$ with a $3 \mathrm{~mm}$ deep spiral groove cut into one side. A $0.5 \mathrm{~m}$ long $\mathrm{Ku}-$ raray $\mathrm{Y} 11$ (200 ppm dopant) wavelength shifting (WLS) fibre with $\sim 1 \mathrm{~mm}$ diameter has been placed in the groove and optically coupled with the scintillator bulk material by the Bicron BC 600 optical cement. One of the fibre ends is polished and connected with a multi-pixel avalanche photodiode. The other end is also polished and mirrored.

The multi-pixel avalanche photodiode with a metalresistor-semiconductor layer structure operating in the limited Geiger mode (known as MRS APD) is produced by the CPTA Company, Moscow. The photodiode has a

1 GERDA Collaboration, GERDA: The Germanium Detector Array for the search of neutrinoless $\beta \beta$ decay of ${ }^{76} \mathrm{Ge}$ at LNGS, Proposal, (http://www.mpi-hd.mpg.de/GERDA)

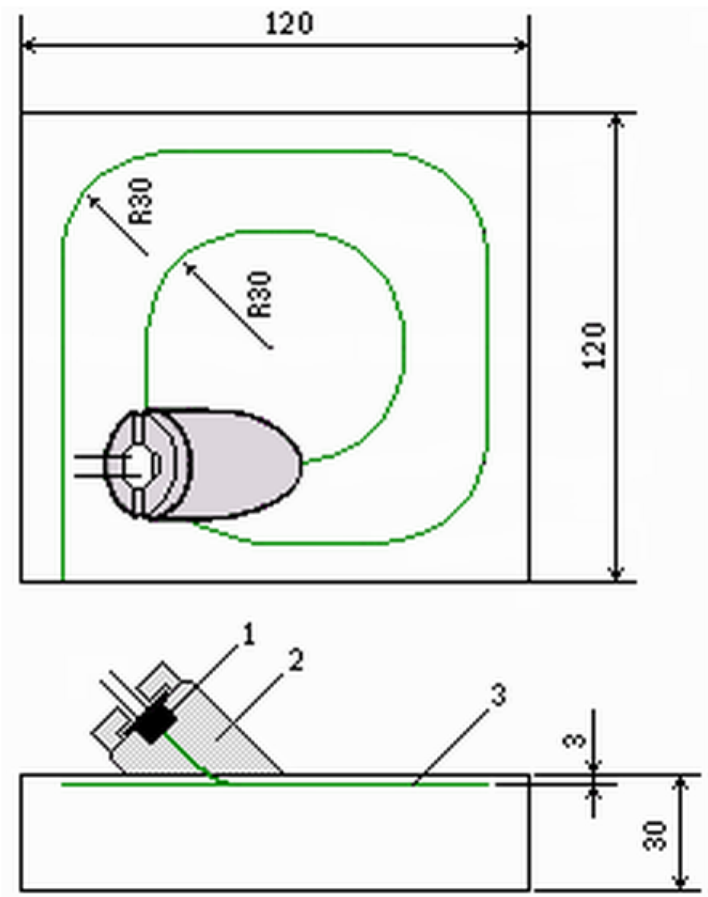

Figure 1. Schematic view of the scintillator counter. 1 - multi-pixel avalanche photodiode MRS APD; 2 - WLS fibre connector; 3 - WLS fibre.

$1.1 \mathrm{~mm}^{2}$ sensitive area with 556 pixels of $45 \times 45 \mu \mathrm{m}^{2}$ each. We refer to [4-6] for more details on MRS APD. The operating voltage of the photodiode used in the measurements was $52.5 \mathrm{~V}$ providing a photon detection efficiency (PDE) of $\sim 20 \%$ at $\lambda=515 \mathrm{~nm}$ at room temperature $\left(22^{\circ} \mathrm{C}\right)$. The dark current counting rate of the photodiode was about 1.2 $\mathrm{MHz}$ above threshold of $\sim 0.5$ photoelectrons (pe). The MRS APD gain was $\sim 5 \times 10^{5}$. Temperature dependencies of the MRS APDs PDE, gain and dark current were thoroughly studied. The temperature coefficients are of $\sim-2 \% /{ }^{\circ} \mathrm{C}$ for the MRS APDs gain and $\mathrm{PDE}$, and $\sim 60 \mathrm{kHz} /{ }^{\circ} \mathrm{C}$ for the the MRS APDs dark current counting rate in the temperature range of $(0 \div 30)^{\circ} \mathrm{C}$.

The charge spectrum of events measured with the scintillator counter in the surface laboratory in a self-triggered mode at room temperature is shown in Fig. 2. The cut off in the left part of the spectrum is due to a discriminator threshold of $\sim 23$ pe. The total counting rate of the counter above this threshold is $\sim 3.7 \mathrm{~Hz}$. A peak produced by cosmic ray muons is clearly seen on the right and corresponds to $\sim 56$ pe. The peak-to-valley ratio of the spectrum is $\sim 2.5$.

The charge spectrum of events measured with the same scintillator counter in the underground laboratory at the depth of 200 m.w.e. is shown in Fig. 3. The ADC pedestal 


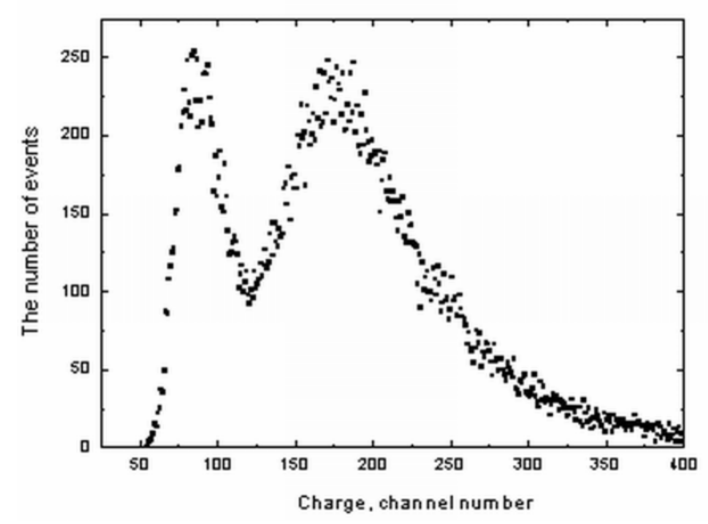

Figure 2. Charge spectrum of events detected with the scintillator counter in the surface laboratory.

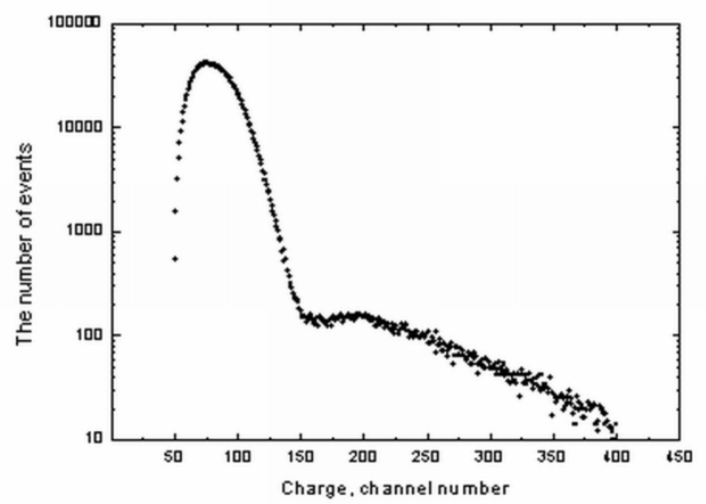

Figure 3. Charge spectrum of events detected with the scintillator counter at the underground laboratory at the depth of 200 m.w.e.

was subtracted in both spectra. The discriminator threshold was the same as in the measurements at the surface. The temperature in the underground laboratory was stabilized to a level of $\sim 14.5 \pm 0.5^{\circ} \mathrm{C}$ throughout the measurements. The total number of counts in the spectrum was approximately $1.75 \times 10^{6}$ within the total time exposure of $1.39 \times 10^{6} \mathrm{~s}$. The largest contribution to the spectrum is from background due to radioactivity in the surrounding rock. The muon peak in the spectrum corresponds to $\sim 61$ pe. The observed shift in the muon peak position in comparison to the spectrum registered in the surface laboratory is due to an increase of the photodiode gain and photon detection efficiency because of the temperature decrease $[4,5]$. As can be seen in Fig. 3, the cosmic ray muons contribution to the total spectrum of the scintillator counter is negligibly small at such threshold but at the same time the muon detection efficiency is sufficiently high. Thus the total counting rate of the scintillator counter was $\sim 1.3 \mathrm{~Hz}$ above the threshold of $\sim 0.37$ MIP and almost entirely due to radioactivity in the rock surrounding the underground laboratory.

\section{Results}

The results of the background counting rate measurement with the scintillator counter presented in the paper will be useful for the EMMA experiment as well as for the other currently running and being planned underground experiments.

\section{Acknowledgements}

The authors would like to thank Dr. V. Ch. Lubsandorzhieva and Dr. D. G. Middleton for careful reading of the manuscript and many valuable remarks and discussions.

\section{References}

[1] G. Angloher et al., arXiv:0809.1829v1

[2] T. Enqvist et al., Nucl. Phys. B-Proc. Sup. 165, 478 (2007)

[3] T. Enqvist et al., Nucl. Phys. B-Proc. Sup. 175-176, 307 (2008)

[4] O. Mineev et al., Nucl. Instrum. Meth. A. 577, 540 (2007)

[5] Yu. Musienko et al., Instrum. Exp. Tech.+ 51, 101 (2008)

[6] Yu. Kudenko et al., Instrum. Exp. Tech.+ PD07, 016 (2007) 